\title{
A Distributed Spectrum Sharing Algorithm in Cognitive Radio Networks
}

\author{
Wei Sun ${ }^{\dagger}$, Tong $\mathrm{Liu}^{\dagger}$, Yanmin $\mathrm{Zhu}^{\dagger}$, Bo $\mathrm{Li}^{\ddagger}$ \\ ${ }^{\dagger}$ Shanghai Jiao Tong University \\ ${ }_{\ddagger}^{\ddagger}$ Hong Kong University of Science and Technology \\ Email: ${ }^{\dagger}\{$ sunwit,liutong25691640,yzhu\}@ $\}$ sjtu.edu.cn, ${ }^{\ddagger}$ bli@ @cse.ust.hk
}

\begin{abstract}
In this paper we study a social welfare maximization problem for spectrum sharing in cognitive radio networks. To fully use the spectrum resource, the licensed spectrum owned by the primary user (PU) can be leased to secondary users (SUs) for transmitting data. We first formulate the social welfare of a cognitive radio network, considering the cost for the primary user sharing spectrum and the utility gained for secondary users transmitting data. The social welfare maximization is a convex optimization, which can be solved by standard methods in a centralized manner. However, the utility function of each secondary user always contains the private information, which leads to the centralized methods disabled. To overcome this challenge, we propose an iterative distributed algorithm based on a pricing-based decomposition framework. It is theoretically proved that our proposed algorithm converges to the optimal solution. Numerical simulation results are presented to show that our proposed algorithm achieves optimal social welfare and fast convergence speed.
\end{abstract}

Index Terms-Decomposition, social welfare maximization, optimization, cognitive radio network.

\section{INTRODUCTION}

With the increasing development of wireless communications, spectrum becomes a more and more limited resource. However, many studies [1] [2] [3] show that spectrum is under utilized in reality. The technology of cognitive radio (CR) [4] provides a promising mechanism for flexible usage of spectrum. The utilization of spectrum can be improved by sharing vacant spectrum with unlicensed users.

We consider a cognitive radio network, which consists of a primary user (PU), the licensed owner of spectrum, and several secondary users without licences. Each secondary user consists of a pair of nodes, a sender and a receiver, which have the demand of transmitting data. To fully utilize spectrum, the primary user can lease its vacant spectrum to secondary users with monetary rewards.

We consider the problem of spectrum sharing among secondary users in a cognitive radio network. A cost is incurred and paid by the primary user for allocating spectrum to secondary users. Each secondary user obtains an amount of utility for transmitting data, which contains its private information. We first formulate the cost and the utilities, respectively. Then, our objective of such a cognitive radio network is to maximize the social welfare, which is defined as the sum of the utility obtained by each secondary user minus the cost paid by the primary user.

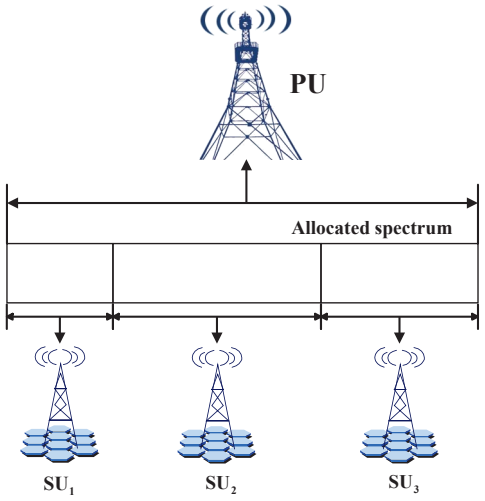

Fig. 1. The illustration of spectrum allocation in a cognitive radio network.

A number of existing works [5] [6] have been done to solve the problem of spectrum sharing in cognitive radio networks. Some of these works [7] [8] [9] take advantage of the models in game theory to characterize cognitive radio networks, which cannot achieve social welfare maximization. These works always assume that secondary users are rational and selfish. Some other works [10] [11] [12] can really maximize the network utility for spectrum sharing. However, the algorithms designed in these works are executed in a centralized manner. Therefore, the privacy of secondary users is not protected. Different with these works, we focus on designing a distributed algorithm to maximize the social welfare.

Such an optimal spectrum sharing problem is significantly challenging because of unique characteristics of cognitive radio networks. First, the utility function of each secondary user contains the user-specific information, which is their privacy. It means that the problem cannot be solved in a centralized manner by the standard methods such as Newton method. A distributed algorithm is necessary by decoupling the problem into several subproblems, each of which can be independently solved by a secondary user. Second, the objective in the problem, maximizing social welfare, is coupled with the cost function of the primary user and the utility function of each secondary user. In other words, the objective function cannot be directly decoupled into several independent subproblems.

In response to the challenges mentioned above, we propose a fully distributed algorithm for spectrum sharing in cognitive 
radio networks. As the shared spectrum is priced by the primary user, we find that the optimal social welfare is obtained when optimal solution of an equivalent pricing problem is achieved. Then, we propose a pricing-based decomposition framework, which decouples the problem into several subproblems. The primary user and each secondary user is responsible for a subproblem, which only contains its own privacy.

Based on the decomposition framework, a distributed algorithm is provided, which is executed iteratively. In each iteration, each secondary user informs the primary user its demanded spectrum, according to the given price. The primary user updates the price based on the collected information. We also propose a price updating rule, under which our iterative algorithm is rigorously proved to converge to the optimal solution in finite steps. Extensive simulations have been conducted to evaluate the social welfare and convergence speed achieved by our distributed algorithm.

We have made three technical contributions in our paper.

- We model the cost paid by the primary user for spectrum sharing and the utility obtained by secondary users. The concept of social welfare is formally defined as the objective of a cognitive radio network to pursue.

- To maximize the social welfare in a distributed manner, we propose a novel pricing-based decomposition framework. A distributed algorithm is designed based on the decomposition framework, where iterative operations are executed by the primary user and the secondary users in turn.

- Rigorous theoretical analysis is conducted to demonstrate that our iterative algorithm converges to the optimal solution in finite steps. Extensive simulation results show that our distributed algorithm can achieve the optimal social welfare, and converge quickly.

The remainder of this paper is organized as follows. We introduce the network model and problem formulation in Section II. The optimal distributed approach is described in Section III. In Section IV, simulation results are reported to show the performance of our algorithm and compared algorithms. We review related work in Section V and conclude the paper in Section VI.

\section{Network Model and Problem Formulation}

In this section, we first describe the model of cognitive radio networks, and then formulate the problem solved in this paper.

\section{A. Network Model}

We consider a cognitive radio network which consists a primary user and $n$ secondary users. The set of secondary users is denoted by $\left\{S U_{1}, S U_{2}, \cdots, S U_{n}\right\}$. Each secondary user actually consists of a sender and a receiver. The primary user, the owner of the spectrum, allocates its unused spectrum to secondary users. Each secondary user is allocated with a portion of spectrum, namely bands. Each band is associated with a bandwidth representing a divided frequency range. The bandwidth allocated to secondary user $S U_{i}$ is noted by $p_{i}, 1 \leq$ $i \leq n$. We assume that the primary user can communicate with each secondary user directly. Moreover, the frequency allocated to different secondary user is orthogonal to avoid interference. The network model is illustrated in Fig. 1.

\section{B. Problem Formulation}

In this subsection, we formulate the social welfare of a cognitive radio network, including two folds: the cost paid by the primary user for spectrum allocation and the utility obtained by the secondary users for data transmission.

Cost for spectrum allocation. The spectrum sharing with secondary users may impact the data transmission of the primary user. This leads to a highly increasing cost associated with the allocated bandwidth, which is formulated as an exponential function. We define the cost function as follows,

Definition 1. The cost function of the primary user is formulated as

$$
C(\mathbf{p})=\beta e^{\sum_{\mathrm{i}=1}^{n} p_{i}}-1
$$

where $\mathbf{p}=\left(p_{1}, p_{2}, \cdots, p_{m}\right)$ and $\beta$ is a system parameter.

The primary user expects a payment in return from secondary users for using spectrum. The primary user provides a price $q_{i}$ for the spectrum spent by the $S U_{i}$. Considering this payment, we formulate the profit of the primary user in Definition 2.

Definition 2. The primary user obtains a profit for sharing spectrum as

$$
\Phi(\mathbf{p}, \mathbf{q})=\mathbf{p q}^{T}-C(\mathbf{p}) .
$$

Utility for data transmission. It is intuitive that each secondary user obtains a utility for transmitting data. According to the bandwidth $p_{i}$ allocated to the secondary user, we define the utility function in the following,

Definition 3. The utility function of secondary user $S U_{i}$ is defined as

$$
u_{i}\left(p_{i}\right)=a_{i} \log \left(1+p_{i}\right),
$$

where $a_{i}$ is a user-specific parameter.

According to the law of diminishing marginal utility in economics [13], the log function implies that the increasing ratio of utility gain obtained by secondary users degrades as the allocated bandwidth increases. Note that the parameter $a_{i}$ is the privacy information of secondary user $S U_{i}$. which is unknown to the primary user and other secondary users.

Thus, the payoff of $S U_{i}$ is formulated as follows,

Definition 4. The payoff of secondary user $S U_{i}$ is equal to the utility obtained minus the payment paid to the primary user,

$$
P_{i}\left(q_{i}, p_{i}\right)=u_{i}\left(p_{i}\right)-q_{i} p_{i} .
$$

In this paper, we consider secondary users are rational, trying to maximize their own payoffs given a price $q_{i}$ by the secondary user.

Social welfare. We introduce the concept of social welfare, which is equal to the utilities gained by secondary users 
minus the cost paid by the primary user, is formulated in the following definition.

Definition 5. The social welfare of a cognitive radio network is defined as

$$
S(\mathbf{p})=\sum_{i=1}^{n} u_{i}\left(p_{i}\right)-C(\mathbf{p}) .
$$

In this paper, we aim to maximize the social welfare of a cognitive radio network. The problem of social welfare maximization is defined in the following.

Definition 6. The problem of maximizing the social welfare of a cognitive radio network is defined as

$$
\begin{array}{lc}
\max & \sum_{i=1}^{n} u_{i}\left(p_{i}\right)-C(\mathbf{p}) \\
\text { s.t. } & p_{i} \in\left[0, \delta_{i}\right], \forall i \in[1, n],
\end{array}
$$

where $\delta_{i}$ is the upper bound of $p_{i}$, determined by secondary user $S U_{i}$.

Note that the problem defined in Definition 6 is a convex optimization problem. According to [14], the problem must have an optimal solution $\mathbf{p}^{*}=\left[p_{1}^{*}, p_{2}^{*}, \cdots, p_{m}^{*}\right]$.

The problem of maximizing the social welfare in a cognitive radio network should be solved in a distributed manner. First, if the problem is centrally solved in one point, it incurs a huge computation load on the single point. Second, the utility function of each secondary user is privacy, which leads to unknowing the exact formulation of social welfare.

\section{Optimal Distributed Approach}

\section{A. Overview}

In response to the difficulties described above, we design a distributed algorithm based on a pricing-based decomposition method. The algorithm can achieve the maximal social welfare and protect the private information for each secondary user at the same time.

We first convert the original problem in (6) into an equivalent pricing problem. The optimal spectrum allocation can be obtained when the optimal prices are achieved. Then, we propose a decomposition framework, which decouples the optimization problem into several subproblems. In the framework, each secondary user maximizes its own payoff based on the price given by the primary user. The primary user updates the price after collecting the returned spectrum demand from secondary users. The above operations are executed iteratively until the spectrum allocation converges to the optimal solution. A price updating rule is also provided, given the insight of the condition that the optimal prices should satisfy. At last, we theoretically prove that our iterative algorithm converges to the optimum in finite steps. The notations appeared in this paper are summarized in Table I.
TABLE I

MAJOR ADOPTED NOTATIONS.

\begin{tabular}{c|l}
\hline Variable & Description \\
\hline \hline$S U_{i}$ & The $i$-th secondary user \\
\hline$n$ & The number of secondary users \\
\hline$p_{i}$ & $\begin{array}{l}\text { The bandwidth of spectrum allocated to the } i \text {-th } \\
\text { secondary user }\end{array}$ \\
\hline$q_{i}$ & The price given to the $i$-th secondary user \\
\hline$\beta$ & $\begin{array}{l}\text { The system parameter in the cost function of the } \\
\text { primary user }\end{array}$ \\
\hline$a_{i}$ & $\begin{array}{l}\text { The user-specific parameter in the utility of the } i \text {-th } \\
\text { secondary user }\end{array}$ \\
\hline$C(\mathbf{p})$ & The cost function of the primary user \\
\hline$\Phi(\mathbf{p}, \mathbf{q})$ & The profit function of the primary user \\
\hline$u_{i}\left(p_{i}\right)$ & The utility function of the $i$-th secondary user \\
\hline$P_{i}\left(q_{i}, p_{i}\right)$ & The payoff function of the $i$-th secondary user \\
\hline$S(\mathbf{p})$ & The social welfare of a cognitive radio network \\
\hline$t$ & The current number of iterations \\
\hline$\tilde{q}_{i}^{(t)}$ & $\begin{array}{l}\text { The price updated for the } i \text {-th secondary user in the } \\
t \text {-th iteration }\end{array}$ \\
\hline$\tilde{p}_{i}^{(t)}$ & $\begin{array}{l}\text { The value of spectrum computed by the } i \text {-th sec- } \\
\text { ondary user in the } t \text {-th iteration }\end{array}$ \\
\hline$\varepsilon$ & The system variable in the price updating rule \\
\hline
\end{tabular}

Algorithm 1 Optimal Distributed Algorithm for Spectrum Sharing

Input: A cognitive radio network consisting of many secondary users, denoted by $\left\{S U_{1}, S U_{2}, \cdots, S U_{n}\right\}$. The cost function of the primary user is $C(\mathbf{p})$ and the utility function of each secondary user is $u_{i}\left(p_{i}\right), \forall i \in\{1, \cdots, n\}$.

Output: The optimal spectrum allocation $p_{i}^{*}$ for each secondary user.

1: $t=0 / / t$ counts the number of iterations.

2: The primary user sets the same initial value $q^{(0)}=\theta$ sent to all secondary users, where $\theta$ is a little positive real value.

3: Repeat for $t=0,1, \cdots$.

4: For each secondary user, the value of $\tilde{p}_{i}^{(t+1)}$ is computed according to

$$
\tilde{p}_{i}^{(t+1)}\left(q_{i}\right)=\underset{p_{i} \in\left[0, \delta_{i}\right]}{\arg \max } u_{i}\left(p_{i}\right)-q_{i} p_{i} .
$$

5: For the primary user, according to the returned $\tilde{p}_{i}^{(t+1)}$, the value of $q_{i}{ }^{(t+1)}$ is updated as

$$
q_{i}^{(t+1)}=(1-\varepsilon) q_{i}^{(t)}+\left.\varepsilon \frac{\partial C(\mathbf{p})}{\partial p_{i}}\right|_{\mathbf{p}=\tilde{\mathbf{p}}^{(t+1)}} .
$$

6: Until if $\left\|\tilde{\boldsymbol{p}}^{(t+1)}-\tilde{\boldsymbol{p}}^{(t)}\right\|_{\infty} \leq \zeta$, where $\zeta$ is a tunable little real number.

7: $\boldsymbol{p}^{*}=\tilde{\boldsymbol{p}}^{(t+1)}, \mathbf{q}^{*}=\mathbf{q}^{(t+1)}$.

\section{B. Equivalent optimal pricing problem}

As mentioned above, the primary user gives a price $q_{i}$ to each secondary user for leasing spectrum. According to the price, each secondary user decides the bandwidth of the spectrum $p_{i}$ it demands. Based on the pricing mechanism, the social welfare can be rewritten as follows, 


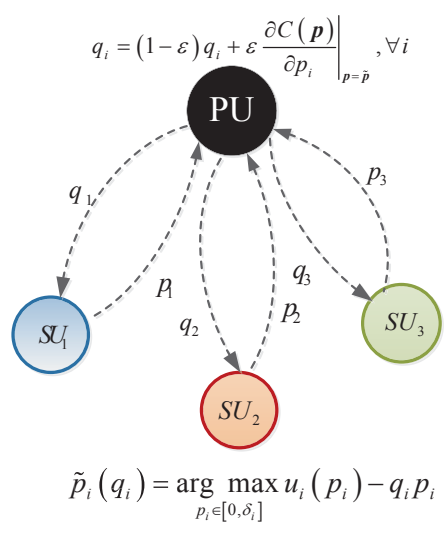

Fig. 2. The pricing-based decomposition framework

$$
\begin{aligned}
S(\mathbf{p}) & =\sum_{i=1}^{n} u_{i}\left(p_{i}\right)-C(\mathbf{p}) \\
& =\sum_{i=1}^{n}\left(u_{i}\left(p_{i}\right)-p_{i} q_{i}\right)+\mathbf{p q}^{T}-C(\mathbf{p}) \\
& =\sum_{i=1}^{n} P_{i}\left(q_{i}, p_{i}\right)+\Phi(\mathbf{p}, \mathbf{q})
\end{aligned}
$$

Considering secondary users are rational, they will choose the bandwidth of allocated spectrum by maximizing their own payoffs given a spectrum price,

$$
\tilde{p}_{i}\left(q_{i}\right)=\underset{p_{i} \in\left[0, \delta_{i}\right]}{\arg \max } u_{i}\left(p_{i}\right)-q_{i} p_{i} .
$$

We denote $\tilde{p}_{i}\left(q_{i}\right)$ as $\tilde{p}_{i}$ for short in the next context. Therefore, the social welfare maximization problem (6) can be transformed into an equivalent optimal pricing problem, which determines the optimal price vector $\mathbf{q}=\left[q_{1}, \ldots, q_{n}\right]$ by maximizing the social welfare as follows,

$$
\max _{\mathbf{q}} \Phi(\tilde{\mathbf{p}}, \mathbf{q})+\sum_{i=1}^{n} P_{i}\left(q_{i}, \tilde{p}_{i}\right)
$$

We illustrate why the pricing problem is equivalent to the original problem briefly. Assume that the optimal solution of problem (10) is $\mathbf{q}^{*}$. The secondary user chooses $\tilde{p}_{i}\left(q_{i}^{*}\right)$ depending on the optimal price $q_{i}{ }^{*}$ by maximizing the payoff (9). After knowing $\tilde{p}_{i}\left(q_{i}^{*}\right)$ and $q_{i}{ }^{*}$, the social welfare is maximized depending on (8). Therefore, the optimal solution of $\mathbf{p}$ can be obtained when finding the optimal price vector $\mathbf{q}^{*}=\left[q_{1}^{*}, \cdots, q_{n}^{*}\right]$.

\section{Pricing-based decomposition framework}

In this subsection, we introduce a pricing-based decomposition framework, which is shown in Fig.2.

Each secondary user computes the value of $\tilde{p}_{i}$ as shown in (9) locally and sends the result to the primary user. According to the collected value of $\tilde{p}_{i}$, the primary user updates the price $q_{i}$ according to a updating rule, which is given in the coming context. The alternate operations are executed iteratively by the primary user and secondary users in turn until the optimal solution $\mathbf{q}^{*}$ and $\mathbf{p}^{*}$ is achieved.

Intuitively, the price updating rule is significant to guarantee the iterative operations eventually converge to the optimal solution. Here, we propose a pricing updating rule according to the the Karuth-Kuhn-Tucker (KKT) condition. According to the convex optimization theory [15], the optimal solution $\mathbf{q}^{*}=\left[q_{1}^{*}, \cdots, q_{n}^{*}\right]$ of (10) must satisfy the KKT condition as shown in the following proposition.

Proposition 1. An optimal price vector $\boldsymbol{q}^{*}=\left[q_{1}^{*}, \cdots, q_{n}^{*}\right]$ should satisfy

$$
q_{i}^{*}=\left.\frac{\partial C(\boldsymbol{p})}{\partial p_{i}}\right|_{\boldsymbol{p}=\tilde{\boldsymbol{p}}}, \forall i
$$

where $\tilde{\boldsymbol{p}}=\left[\tilde{p}_{1}, \cdots, \tilde{p}_{n}\right]^{T}$ with $\tilde{p}_{i}=\tilde{p}_{i}\left(q_{i}^{*}\right)$ given in (9).

Proof: First, according to the first order condition, there exists $\partial P_{i}\left(p_{i}, q_{i}\right) / \partial p_{i}=0$ for (2). Therefore, we obtain

$$
q_{i}=u_{i}^{\prime}\left(\tilde{p}_{i}\right) \text {. }
$$

Moreover, applying the KKT condition $\partial S(\mathbf{p}) / \partial q_{i}=0$ to (5), there is $\left(u_{i}^{\prime}\left(\tilde{p}_{i}\right)-\left.\frac{\partial C(\mathbf{p})}{\partial p_{i}}\right|_{\mathbf{p}=\tilde{\mathbf{p}}}\right) \frac{\partial \tilde{p}_{i}}{\partial q_{i}}=0$ since $\frac{\partial \tilde{p}_{i}}{\partial q_{i}}<0$, we have

$$
u_{i}^{\prime}\left(\tilde{p}_{i}\right)-\left.\frac{\partial C(\mathbf{p})}{\partial p_{i}}\right|_{\mathbf{p}=\tilde{\mathbf{p}}}=0 .
$$

The proposition is proved by combining the (12) and (13).

Given the insight of Proposition 1, we propose a price updating rule as follows,

$$
q_{i}=(1-\varepsilon) q_{i}+\left.\varepsilon \frac{\partial C(\mathbf{p})}{\partial p_{i}}\right|_{\mathbf{p}=\tilde{\mathbf{p}},} \forall i,
$$

where $\varepsilon \in(0,1]$ is a tunable parameter.

Note that in our decomposition framework, updating price is unnecessary to be executed after all $\tilde{p}_{i}, \forall i \in\{1, \cdots, n\}$ are returned. The primary user can update $q_{i}$ for secondary user $S U_{i}$ arbitrary times before $q_{j}(j \neq i)$ is updated. It means that our decomposition framework can be realized in an asynchronous way so that the number of iterations can be largely degraded as different secondary users have different communication delay with the primary user.

\section{Distributed Algorithm}

In this subsection, we propose a distributed algorithm to maximize the social welfare of a cognitive radio network. In the distributed algorithm, the primary user and all secondary users should participate as follows,

- For each secondary user $S U_{i}$, it maximizes the payoff $P_{i}\left(p_{i}, q_{i}\right)=u_{i}\left(p_{i}\right)-q_{i} p_{i}$ locally to obtain the value of $\tilde{p}_{i}$ based on the price $q_{i}$ given by the primary user. Each secondary user only needs to know its own utility function.

- For the primary user, it updates the value of $q_{i}$ based on $\tilde{p}_{i}$ returned from each secondary user. The price updating rule is given in (14). 
The primary user generates a new price based on an original price and partial derivative $\left.\frac{\partial C(\mathbf{p})}{\partial p_{i}}\right|_{\mathbf{p}=\tilde{\mathbf{p}}}$, which pushes the price $q_{i}$ towards the optimal value $q_{i}^{*}=\frac{\partial C\left(\mathbf{p}^{*}\right)}{\partial p_{i}}$. The details of the distributed algorithm are described in algorithm 1.

\section{E. Convergence Analysis}

Contraction Mapping. Many iterative algorithms can be expressed as $x(l+1)=\varphi(x(l)), l=0,1, \cdots$, where $x(\cdot) \in X$ and $l$ denotes the number of iterations. Mapping $\varphi$ is called a contraction if

$$
\|\varphi(x)-\varphi(y)\| \leq \kappa\|x-y\|, \forall x, y \in X,
$$

where $\|\cdot\|$ is a norm and $\kappa \in[0,1)$ is called a modulus of $\varphi$. Moreover, the mapping $\varphi$ is called a pseudo-contraction if there exists a fixed point $x^{*} \in X$ (means $x^{*} \in \varphi\left(x^{*}\right)$ ) and

$$
\left\|\varphi(x)-x^{*}\right\| \leq\left\|x-x^{*}\right\|, \forall x \in X .
$$

The convergence property of contraction or pseudocontractions is given in Theorem 1.

Theorem 1 (Geometric Convergence). Supposing the mapping or the pseudo-contraction and the modulus of $\varphi$ is $\kappa \in[0,1)$. Then, $\varphi$ has an unique fixed point $x^{*}$ and a sequence $\{x(l), l=0,1, \cdots\}$ generated by $x(l+1)=\varphi(x(l))$ satisfies

$$
\left\|x(l)-x^{*}\right\| \leq \kappa^{l}\left\|x(0)-x^{*}\right\|, \forall l \geq 0,
$$

for every choice of initial $x(0) \in X$. In particular, $x(l)$ converges to $x^{*}$ geometrically.

In this section, we analyze the convergence of our algorithm under the condition of $\varepsilon=1$ and $\varepsilon<1$ in Proposition 2 and Proposition 3, respectively. For simplicity, the second order partial derivatives of $C(\mathbf{p})$ is denoted by $\partial_{p_{j} p_{i}} C(\mathbf{p})=\frac{\partial C(\mathbf{p})}{\partial p_{j} \partial p_{i}}$.

Convergence of Algorithm 1 with $\varepsilon=1$. We first define a notation $[\lambda]_{i}^{+}$to denote the projection of $\lambda_{i} \in \Re$ onto the range $\left[0, \tau_{i}\right]$,

$$
[\lambda]_{i}^{+}=\arg \max _{z=\left[0, \tau_{i}\right]}\left|z-\lambda_{i}\right| .
$$

Briefly, a solution to (9) is equivalent to

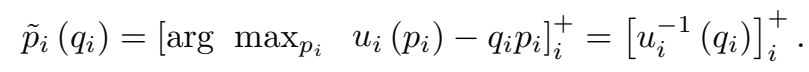

Therefore, when $\varepsilon=1$, the price updating rule turns to $q_{i}=c_{i}(\tilde{\mathbf{p}})$. Substituting it into $\tilde{p}_{i}\left(q_{i}\right)$ obtains

$$
\tilde{p}_{i}=\varphi_{i}(\tilde{\mathbf{p}})=\left[u_{i}^{-1}\left(c_{i}(\tilde{\mathbf{p}})\right)\right]_{i}^{+} .
$$

Algorithm 1 applied with $\varepsilon=1$ eventually achieves convergence under a certain condition which is given in the following proposition.

Proposition 2. Supposing $\varepsilon=1$, if we have

$$
\sum_{j=1}^{n}\left|\partial_{p_{j} p_{i}} C(\mathbf{p})\right|<\min _{p_{i}}\left|u_{i}^{\prime}\left(p_{i}\right)\right|, \forall \mathbf{p} \in \prod_{i}\left[0, \delta_{i}\right],
$$

$\left\{\tilde{p}_{i}\left(q_{i}^{(t)}\right)\right\}$ generated by Algorithm 1 converges geometrically [16] to the optimal solution $p_{i}^{*}$ of (6), giving an initial value $q_{i}^{(0)}$.

Proof: For each $i$, a function $g_{i}$ is defined as follows.

$$
g_{i}(r)=u_{i}^{-1}\left(c_{i}(z(r))\right)=u_{i}^{-1}\left(c_{i}(r x+(1-r) y)\right),
$$

where $g_{i}(r)$ is differentiable and $r \in[0,1]$. We have

$$
\begin{aligned}
\left\|\varphi_{i}(x)-\varphi_{i}(y)\right\| & =\left|\left[u_{i}^{-1}\left(c_{i}(x)\right)\right]\right|_{i}^{+}-\left|\left[u_{i}^{-1}\left(c_{i}(y)\right)\right]\right|_{i}^{+} \\
& \leq\left|g_{i}(1)-g_{i}(0)\right|=\left|\int_{0}^{1} \frac{d g_{i}(r)}{d r}\right| \\
& \leq \int_{0}^{1}\left|\frac{d g_{i}(r)}{d r}\right| \leq \max _{r \in[0,1]}\left|\frac{d g_{i}(r)}{d r}\right|,
\end{aligned}
$$

where the first inequality is because $\left|\left[x_{i}\right]_{i}^{+}-\left[y_{i}\right]_{i}^{+}\right| \leq$ $\left|x_{i}-y_{i}\right|$ for all $x_{i}-y_{i} \in \Re$. Furthermore, applying a chain rule, we have

$$
\begin{aligned}
& \frac{d g_{i}(r)}{d r}=\left|\sum_{j=1}^{n} \nabla_{j} c_{i}^{-1}\left(c_{i}(r x+(1-r) y) \cdot\left(x_{j}-y_{j}\right)\right)\right| \\
& \quad \leq\left|\left(u_{i}^{-1}\right)^{\prime}\left(c_{i}(z(r))\right)\right| \cdot \sum_{j=1}^{n}\left|\nabla_{j} c_{i}(z(r))\right| \cdot\left|x_{j}-y_{j}\right| .
\end{aligned}
$$

If condition (19) holds, we have

$$
\sum_{j=1}^{n}\left|\nabla_{j} c_{i}(x)\right|<\min _{x_{i}}\left(u^{\prime}{ }_{i}\left(x_{i}\right)\right) \leq\left|u_{i}^{\prime}\left(u_{i}^{-1}\left(c^{\prime}{ }_{i}(x)\right)\right)\right|
$$

for all $x \in \prod_{i}\left[0, \tau_{i}\right]$. Therefore, there exists a number $\kappa<1$ which enables that

$$
\left|\frac{d g_{i}(r)}{d r}\right| \leq \kappa \max _{j}\left|x_{j}-y_{j}\right|=\kappa\|x-y\|_{\infty}, \forall r \in[0,1]
$$

Therefore, we have

$$
\left\|\varphi_{i}(x)-\varphi_{i}(y)\right\|_{\infty} \leq \kappa\|x-y\|_{\infty}, \forall x, y \in \prod_{i}\left[0, \tau_{i}\right],
$$

which shows $\varphi_{i}$ is a contraction with modulus $\kappa$ respect to a maximum norm. Therefore, a proposition is proved.

Convergence of Algorithm 1 with $\varepsilon<1$. We relax a value of $\varepsilon$ by considering a convergence condition with $\varepsilon<1$. In this situation, we suppose that $\tilde{\mathbf{p}}\left(\mathbf{q}^{(l)}\right)$ generated with each iteration $l$ is always within a range $\prod_{i}\left[0, \tau_{i}\right]$. Therefore, we can rewrite a solution to (9) as $\tilde{p}_{i}\left(q_{i}\right)=u_{i}^{-1}\left(q_{i}\right)$, which has a little difference from $\varepsilon=1$ situation. Combining it with $q_{i}=(1-\varepsilon) q_{i}+\varepsilon c_{i}(\tilde{\mathbf{p}})$, we can obtain

$$
\tilde{p}_{i}=\varphi_{i}(\tilde{\mathbf{p}})=u_{i}^{-1}\left((1-\varepsilon) u_{i}\left(\tilde{p}_{i}\right)+\varepsilon c_{i}(\tilde{\mathbf{p}})\right)
$$

Applied with (20), the Algorithm 1 can achieve optimal under a convergence condition in the following proposition.

Proposition 3. Set $\tilde{p}_{i}\left(q_{i}^{(0)}\right)$ to be 0 for all $i$ or $\delta_{i}$ for all $i$. If we have

$$
\left\{\begin{array}{c}
0 \leq \varepsilon \leq\left(1+\frac{\partial_{p_{j} p_{i}} C(\mathbf{p})}{c^{\prime} i\left(p_{i}\right)}\right)^{-1} \\
\sum_{j \neq i} \partial_{p_{j} p_{i}} C(\mathbf{p}) \geq 0
\end{array}, \forall \mathbf{p} \in \prod_{i}\left[0, \delta_{i}\right],\right.
$$

$\left\{\tilde{p}_{i}\left(q_{i}^{(t)}\right)\right\}$ generated by our algorithm 1 converges geometrically [16] to the optimal solution $p_{i}^{*}$ of (6). 
Proof: For each $i$ a function $g_{i}$ is defined as follows.

$$
g_{i}(r)=u_{i}^{-1}\left((1-\varepsilon) u_{i}(z(r))\right)+\varepsilon c_{i}(z(r)),
$$

where $z(r)=r x+(1-r) y$. have

Supposing $x<x^{*}$, we will show $x_{i}<\varphi_{i}(x) \leq x_{i}^{*}$. We

$$
\varphi_{i}(x)-\varphi_{i}\left(x^{*}\right)=g_{i}(1)-g_{i}(0)=\int_{0}^{1} \frac{d g_{i}(r)}{d r} d r,
$$

where $\frac{d g_{i}(r)}{d r}$ is given by (22).

$$
\begin{aligned}
& \frac{d g_{i}(r)}{d r}=\left(u_{i}^{-1}\right)^{\prime}\left((1-\varepsilon) u_{i}(z(r))+\varepsilon c_{i}(z(r))\right) \\
& \cdot(1-\varepsilon) u_{i}^{\prime}(z(r)) \cdot\left(x_{i}-x_{i}^{*}\right)+\varepsilon \sum_{j} \nabla_{j} c_{i}(z(r)) \cdot\left(x_{j}-x_{j}^{*}\right) \\
& =\left(u_{i}^{\prime}\left(\varphi_{i}(z(r))\right)\right)^{-1} \cdot \\
& \left(\begin{array}{l}
\left((1-\varepsilon) u_{i}^{\prime}(z(r))+\varepsilon \nabla_{i} c_{i}(z(r))\right) \cdot\left(x_{i}-x_{i}^{*}\right) \\
+\varepsilon \sum_{j \neq i} \nabla_{j} c_{i}(z(r)) \cdot\left(x_{j}-x_{j}^{*}\right)
\end{array}\right)
\end{aligned}
$$

Applying $h_{i}^{-1}(\cdot)$ to both sides yields $x_{i}<\varphi_{i}(x)$. Therefore, there exists a number $\kappa<1$ enables that $\left|\varphi_{i}(x)-x_{i}^{*}\right| \leq$ $\kappa\left|x-x^{*}\right|$, which is equivalent to

$$
\left\|\varphi_{i}(x)-x^{*}\right\|_{\infty} \leq \kappa\left\|x-x^{*}\right\|_{\infty}, \forall x_{i} \in\left[0, x_{i}^{*}\right] .
$$

In other words, $\varphi_{i}$ is a pseudo-contraction in $\left[0, x_{i}^{*}\right]$. Similarly, if $x>x^{*}$, we can get $\varphi_{i}$, which is a pseudo-contraction in $\left[x_{i}^{*}, \tau_{i}^{*}\right]$. Therefore, the proposition is proved.

\section{Performance Evaluation}

\section{A. Methodology and Simulation Setup}

We perform simulations to evaluate the performance of our optimal distributed algorithm, compared with a baseline algorithm as follows.

Distributed weighted allocation scheme (DWAS). First, the primary user provides an initial value $q_{0}$ for all secondary users. Each secondary user $S U_{i}$ returns the value of $\widetilde{p}_{i}\left(q_{0}\right)$ by maximizing $P\left(q_{i}, p_{i}\right)$. Next, the primary user decides the final value of $q_{i}, \forall i \in\{1, \cdots, n\}$ by maximizing $\Phi(\mathbf{q}, \boldsymbol{p})$, assuming that $u_{i}\left(p_{i}\right)$ is a linear function of $p_{i}$, i.e., $u_{i}\left(p_{i}\right)=$ $b_{i} p_{i} . b_{i}$ represents the weight of secondary user $S U_{i}$, which is computed as $\widetilde{p}_{i}\left(q_{0}\right) / q_{0}$. After receiving $q_{i}$, each secondary user computes its optimal $\widetilde{p}_{i}\left(q_{i}\right)$.

In addition to the DWAS, we also compare our algorithm with the optimal value. The optimal value is obtained by maximizing the social welfare directly.

The evaluation of our algorithm is performed from two aspects, including the network performance and the convergence speed. The metrics of network performance are the social welfare and the total allocated spectrum. We perform the simulations with varying two factors: the number of secondary users and the value of $\beta$. The metric of convergence speed is the number of iterations. The simulations are performed under different numbers of secondary users and values of $\varepsilon$. The initial values of $q_{i}, \forall i \in\{1,2, \cdots, n\}$ in our proposed algorithm and the DWAS are set to 1 . The default values of $\beta$ and $\varepsilon$ are 0.2 and 0.1 , respectively. The values of $a_{i}$ are standard uniformly distributed in the open interval $(0.5,1)$.

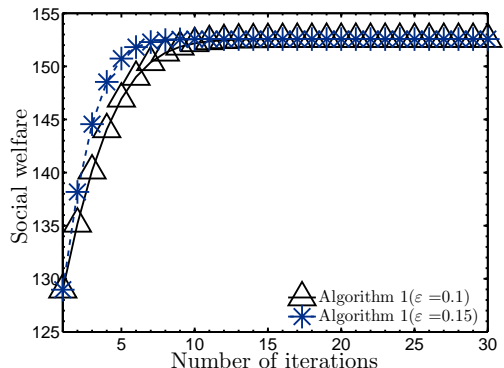

Fig. 3. Social welfare achieved with the number of iterations

\section{B. Impacts of the number of secondary users}

We first study the social welfare and allocated spectrum of our algorithm and the DWAS under the condition of different numbers of secondary users, compared with the optimum. The results are shown in Fig. 4 and Fig. 7.

Fig. 4 shows that the social welfare obtained by our algorithm performs as well as the optimum, and much better than the DWAS. The social welfare obtained by our algorithm and the DWAS increases with the increasing number of secondary users. When there are 20 secondary users, the social welfare obtained by our algorithm is $50.34 \%$ higher than the DWAS.

In Fig. 7, the spectrum allocated by our algorithm is the same as the optimum, and more than the DWAS. The spectrum obtained by our algorithm and the DWAS increases with the increasing number of secondary users. However, the spectrum allocated by DWAS is always less than the spectrum allocated by our algorithm. When there are 20 secondary users, the allocated spectrum obtained by our algorithm is $96.23 \%$ more than the DWAS.

\section{Effects of the value of $\beta$}

We next study the performance of our algorithm and the DWAS under the condition of different values of $\beta$, compared with the optimum. The results are shown in Fig. 5 and Fig. 8.

Fig. 5 shows that the social welfare achieved by our algorithm performs as well as the optimum, and more than the DWAS. The social welfare obtained by our algorithm and the DWAS decreases with the increasing value of $\beta$. When there are 20 secondary users, the social welfare obtained by our algorithm is $40 \%$ more than the DWAS.

In Fig. 8, the spectrum allocated by our algorithm is the same as the optimum, and more than the DWAS. For our algorithm and the DWAS, the allocated spectrum decreases, when the value of $\beta$ increases. When there are 20 secondary users, the allocated spectrum obtained by our algorithm is $97.43 \%$ more than the DWAS.

\section{Convergence Speed}

In this subsection, we study the convergence speed of our algorithm under different numbers of secondary users and values of $\varepsilon$. The results are shown in Fig. 3, Fig. 6 and Fig. 9. 

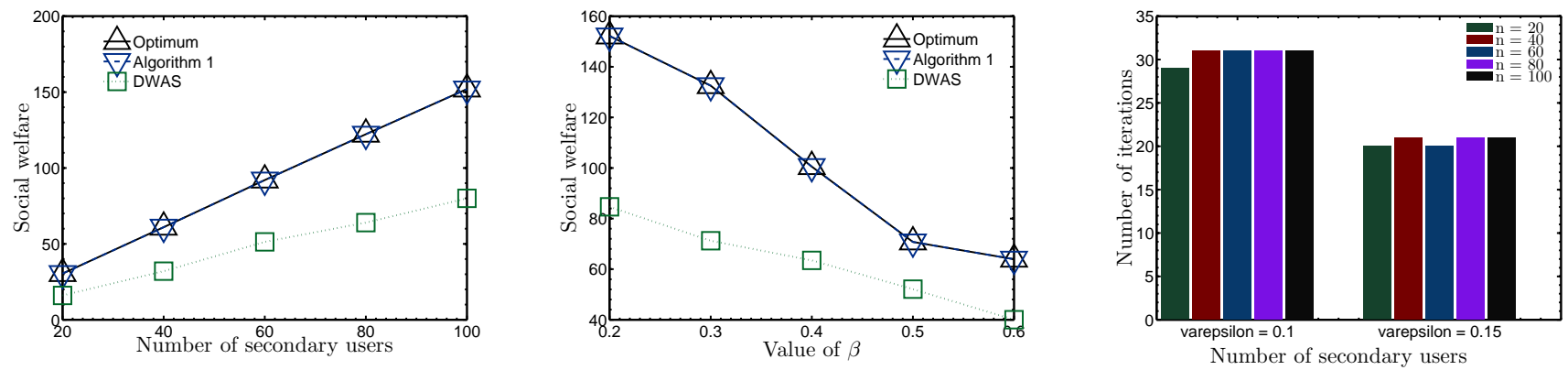

Fig. 4. Social welfare comparison of different Fig. 5. Social welfare comparison of different Fig. 6 . Convergence of Algorithm 1 with different algorithms with varying number of secondary users algorithms with varying $\beta$ number of secondary users
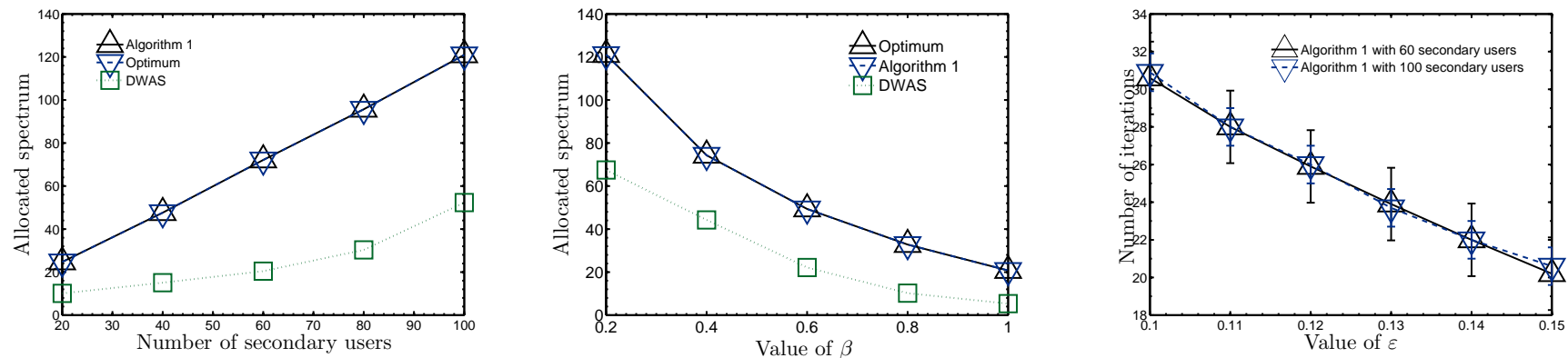

Fig. 7. Allocated spectrum comparison of different Fig. 8. Allocated spectrum comparison of different Fig. 9. Convergence of Algorithm 1 with different algorithms with varying number of secondary users algorithms with varying $\beta$ value of parameter $\varepsilon$

Fig. 3 plots the social welfare achieved by our algorithm in each iteration, when the value of system parameter $\varepsilon$ is 0.1 and 0.15 , respectively. Under the two settings, we can see that 99.9\% of the optimal social welfare is achieved in the first ten iterations, which implies our algorithm converges quickly.

In Fig. 6, We study simulations of our algorithm when the number of secondary users is $20,40,60,80$ and 100 , respectively. The value of parameter $\varepsilon$ is 0.1 and 0.15 . The numbers of iterations under different numbers of secondary users and different values of $\varepsilon$ are close, which illustrates our algorithm is adapted to large-scale cognitive radio networks.

Fig. 9 shows that the convergence speed of our algorithm under the condition of the value of $\varepsilon$ varying from 0.1 to 0.15 . The number of secondary users is 60 and 100. The number of iterations under different values of $\varepsilon$ is less than 32 . The higher value of system parameter $\varepsilon$, the faster the convergence speed. Our algorithm converges fast under varying values of $\varepsilon$.

\section{RELATED WORK}

Increasing works focus on the problems in cognitive radio networks. Some works focus on estimation of interference temperature of the radio environment and detection of spectrum holes. such as [17] [18]. Works in [19] [20] [21] focus on estimation of channel-state information and prediction of channel capacity for using by the transmitter. Some other works [5] [6] focus on transmit-power control and dynamic spectrum management.

The spectrum allocation in cognitive radio networks has been extensively studied. By taking advantage of the models in game theory, some of existing works solve the spectrum sharing problem by achieving Nash Equilibrium. Niyato et al. [7] formulate the problem of spectrum sharing as an oligopoly market competition and use a Cournot game to obtain the spectrum allocation. However, the static Cournot game only adapts to some special environment. Han et al. [9] propose a correlated equilibrium concept for users to have the distributive opportunistic spectrum access. Wang et al. [8] propose a competitive spectrum-sharing scheme according to auction theorem without achieving social welfare maximization.

Some centralized algorithms in some other works can actually achieve the social welfare maximization. However, these algorithms could not protect the privacy of each user. Ileri et al. [12] propose a framework under the regulation of a spectrum policy server for spectrum sharing. Peng et al. [10] propose a framework that defines the spectrum sharing problem for some definitions of overall system utility. The central sever is designed for allocation assignments. Raman et al. [11] propose a centralized spectrum server that coordinates users to sharing a common spectrum. 


\section{CONCLUSION}

The problem of maximizing social welfare for spectrum sharing in cognitive radio networks is studied in this paper. We first define the concept of social welfare by formulating the cost of the primary user and the utility of each secondary user. To protect the private information of secondary users, we propose a distributed algorithm based on a pricing-based decomposition framework. The distributed algorithm is executed iteratively by the primary user and each secondary user in turn. Theoretical analysis is conducted to demonstrate that the algorithm converges to the optimal solution. Extensive simulation results show that our proposed achieves maximal social welfare and converges quickly.

\section{REFERENCES}

[1] P. Gupta and P. R. Kumar, "The capacity of wireless networks," Information Theory, IEEE Transactions on, vol. 46, no. 2, pp. 388-404, 2000 .

[2] J. M. III and Q. G. J. Maguire, "Cognitive radio: making software radios more personal," Personal Communications, IEEE, vol. 6, no. 4, pp. 13$18,1999$.

[3] S. Haykin, "Cognitive radio: brain-empowered wireless communications," Selected Areas in Communications, IEEE Journal on, vol. 23, no. 2, pp. 201-220, 2005.

[4] Q. Zhao and B. M. Sadler, "A survey of dynamic spectrum access," Signal Processing Magazine, IEEE.

[5] W. Yu, "Competition and cooperation in multi-user communication environments," Ph.D. dissertation, 2002.

[6] J. Zander, S. Kim, M. Almgren, and O. Queseth, Radio resource management for wireless networks. Artech House, Inc., 2001.

[7] a. E. H. D. Niyato, "A game-theoretic approach to competitive spectrum sharing in cognitive radio networks," in Wireless Communications and Networking Conference, 2007.
[8] X. Wang, Z. Li, P. c. Xu, Y. y. Xu, X. b. Gao, and H. H. Chen, "Spectrum sharing in cognitive radio networksłan auction-based approach," Systems, Man, and Cybernetics, Part B: Cybernetics, IEEE Transactions on, vol. 40, no. 3, pp. 587-596, 2010.

[9] Z. Han and a. K. R. L. C. Pandana, "Distributive opportunistic spectrum access for cognitive radio using correlated equilibrium and no-regret learning," in Wireless Communications and Networking Conference, 2007.

[10] C. Peng, H. t. Zheng, and B. Y. Zhao, "Utilization and fairness in spectrum assignment for opportunistic spectrum access," Mobile Networks and Applications, vol. 11, no. 4, pp. 555-576, 2006.

[11] C. Raman, R. Yates, and N. B. Mandayam, "Scheduling variable rate links via a spectrum server," in New Frontiers in Dynamic Spectrum Access Networks, 2005.

[12] O. Ileri, D. Samardzija, and N. B. Mandayam, "Demand responsive pricing and competitive spectrum allocation via a spectrum server," in New Frontiers in Dynamic Spectrum Access Networks, 2005.

[13] A. Chateauneuf and M. Cohen, "Risk seeking with diminishing marginal utility in a non-expected utility model," Journal of Risk and Uncertainty, vol. 9, no. 1, pp. 77-91, 1994.

[14] S. P. Boyd and L. Vandenberghe, Convex optimization. Cambridge university press, 2004.

[15] D. P. Bertsekas, A. Nedi, and A. E. Ozdaglar, Convex analysis and optimization. Athena Scientific Belmont, 2003.

[16] K. H. Khalil, Nonlinear systems. Prentice hall Upper Saddle River, 2002, vol. 3 .

[17] S. Haykin, "Communication systems," New York, 2001.

[18] J. D. Thomson, "Spectrum estimation and harmonic analysis," Proc. IEEE INFOCOM, vol. 70, no. 9, pp. 1055-1096, 1982.

[19] S. Haykin, K. Huber, and Z. Chen, "Bayesian sequential state estimation for mimo wireless communications," Proc. IEEE INFOCOM, vol. 92, no. 3, pp. 439-454, 2004.

[20] B. M. Hochwald, T. L. Marzetta, and V. Tarokh, "Multiple-antenna channel hardening and its implications for rate feedback and scheduling," Information Theory, IEEE Transactions on, vol. 50, no. 9, pp. 18931909, 2004

[21] G. J. Foschini and M. J. Gans, "On limits of wireless communications in a fading environment when using multiple antennas," Wireless personal communications, vol. 6, no. 3, pp. 311-335, 1998. 